Review

\title{
A Review on Epidemiology and Thalassemia Distribution, in Relevance to United Arab Emirates Population
}

\author{
${ }^{1}$ Pooja Shivappa, ${ }^{2}$ Grisilda Vidya Bernhardt, ${ }^{3}$ Manal Munir Mufti and ${ }^{3}$ Ayesha Banu \\ ${ }^{l}$ Department of Basic Sciences, RAK Medical and Health Sciences University, UAE \\ ${ }^{2}$ Department of Biochemistry, RAK Medical and Health Sciences University, UAE \\ ${ }^{3} R A K$ Medical College, RAK Medical and Health Sciences University, UAE
}

\author{
Article history \\ Received: 28-03-2021 \\ Revised: 22-04-2021 \\ Accepted: 25-05-2021 \\ Corresponding Author: \\ Pooja Shivappa \\ Department of Basic Sciences, \\ RAK Medical and Health \\ Sciences University, UAE \\ Email: pooja@rakmhsu.ac.ae
}

\begin{abstract}
Thalassemia is a blood disorder passed down through families (inherited) in which the body makes an abnormal form or inadequate amount of hemoglobin. Hemoglobin is the protein in red blood cells that carries oxygen. The disorder results in large numbers of red blood cells being destroyed, which leads to anemia. Depending on the type of mutated globin, there are two types of thalassemia: Alpha- $(\alpha-)$ thalassemia and beta- $(\beta-)$ thalassemia. It is estimated that around 9 million people suffering from thalassemia become pregnant every year worldwide, of which 1.33 million are at risk of developing severe thalassemia disorder. Thalassemia is a problem in the health sector in many Middle East and North Africa (MENA), region Southeast Asia, Central Asia and the Indian subcontinent. Countries in the MENA region, particularly the Gulf Cooperation Council (GCC) countries and the UAE, have higher prevalence of $\beta$-thalassemia and carriers than $\alpha$-thalassemia. The UAE has the most prevalence of thalassemia carrier among all the MENA countries, due to a huge rate of consanguineous marriage. The UAE government has initiated various measure to reduce the incidences of thalassemia among which one such program included launching of a national campaign in 2008 to promote premarital screening that provides non-instructive genetic counseling to non-at-risk couples. Despite various measures of the thalassemia premarital screening program, the premarital screening program has not been able to effectively reduce the frequency of marriages in high-risk couples in UAE. The study concludes that targeting young people and raising awareness of thalassemia and increasing its burden may reduce inter-carrier marriage and reduce the incidence of such disorders.
\end{abstract}

Keywords: Thalassemia, Genetic disorders, MENA region

\section{Introduction}

A genetic blood disorder, Thalassemia produces in the body an abnormal form of hemoglobin. Based on the type of mutated globin, there are two types of thalassemia: Alpha- $(\alpha-)$ thalassemia and beta- $(\beta-)$ thalassemia. When any of the four $\alpha$-globin genes are damaged or altered, $\alpha$ Thalassemia occurs. When both $\beta$-globin genes are damaged or mutated $\beta$-thalassemia occurs (Kim and Tridane, 2017). It can happen that a child received one defective globin genes from each parent, thus two defective globin genes. This is when Major thalassemia occurs. Sometimes a child received a defective gene from only one parent. This is when juvenile Thalassemia occurs. (Cao and Galanello, 2010; Danjou et al., 2011). Marriage is an obvious risk factor for Thalassemia.
Marriage referred to here, is a marriage between two people in a relationship, such as with a second cousin, with an ingestion coefficient greater than 0.0156. To predict the ratio of loci at which an extended family member's child is expected to receive similar copies of the gene from both parents, a method called inbreeding rate is used were the ratio of loci of the concerned occurrence is determined.

People with thalassemia minor can lead healthy and normal lives without treatment because they are asymptomatic. On the other hand, people who suffer from thalassemia major begin with anemia in childhood that prolongs to the life time. Due to red blood cell abnormalities, such cases require regular blood transfusions. Blood transfusion is the recommended treatment (Kim and Tridane, 2017). Homozygous $\alpha$ - 
thalassemia happens when all four $\alpha$-globin genes are deleted. The phenotype of $\beta$-Thalassemia is not homogeneous (heteregenous), ranging from major thalassemia that is transfusion dependent to intermediate forms of mild thalassemia. People having $\beta$-thalassemia major suffer anemia that is very sever and overgrowth of the hepatic spleen and if children with this disease are left untreated, development is delayed and life expectancy is reduced (Danjou et al., 2011). $\alpha$-Thalassemia disorder was previously considered harmless. Although, homozygous $\alpha$-thalassemia, studies show now, is fatal and common in the global population (Kim and Tridane, 2017; Vichinsky, 2005). Hb Barts are formed in the uterus during Homozygous $\alpha$-thalassemia. Hb Bart have very high affinity thus supplies low levels of oxygen to the fetal tissue as a result of which severe hypoxia, heart failure and fetal death in the uterus is caused (Vichinsky, 2005; Weatherall, 2010a). In homozygous $\alpha$-thalassemia, fetal death can be prevented by intrauterine transfusion after initial detection, but it is necessary that transfusion therapy continues (Vichinsky, 2005). Treatment of thalassemia requires large amounts of blood. This treatment consumes large amounts of blood in lieu of other treatments requiring blood transfusions, leading to serious complications that include iron overload, bone deformity and heart disease from infection (Vichinsky, 2005; Weatherall, 2010b).

\section{Epidemiology of Thalassemia}

Kim and Tridane (2017) assessed the presence of thalassemia major among the populations in the Middle East and North Africa (MENA region) due to consanguineous marriages (a significant factor of risk in the case of major thalassemia) and reported that the Kingdom of Saudi Arabia (KSA) has a higher incidences of $\beta$-thalassemia among which the presence of carriers of $\beta$-thalassemia is $1-15 \%$ and $\alpha$-thalassemia carriers is $5-10$. 3 to $5.9 \%$ of prevalence was found in Jordan for $\beta$-thalassemia carriers and 2 to $3.5 \%$ for $\alpha$ thalassemia carriers in the same region. Presence $\beta$ thalassemia carriers of $4.5 \%$ was found in Egypt and a range of 5 to $10 \%$ of incidence of $\alpha$-thalassemia in Kuwait. The prevalence of $\alpha$-thalassemia and $\beta$ thalassemia carriers in the UAE was higher than in Bahrain. The comparison of UAEvsBahrain in prevalence of $\beta$-thalassemia and $\alpha$-thalassemia is 8.5 Vs. $2.9 \%$ and 49 Vs. 24.2\%, respectively (Kim and Tridane, 2017).

Modell and Darlison (2008) study found that the estimated incidence of thalassemia was $0.06 \%$ (per 1000 people) in the US population of 850 million, $0.07 \%$ in the African population of 585 million and $0.13 \%$ in the European population of 879 million, $0.66 \%$ in the South East Asia's population of 1564 million, $0.76 \%$ in the Western Pacific population of 1.761 million. The total number of patients affected with Thalassemia worldwide is estimated to be $0.46 \%$ of the total population of 6217 million (Modell and Darlison, 2008).

$\beta$-Thalassemia major causes severe anemia, which in case of children below 3 years of age, can lead to death. Such death from thalassemia major can be prevented by regular blood transfusions (Modell and Darlison, 2008). In KSA (Kingdom of Saudi Arabia), Ankra-Badu et al. (2001) reported blood transfusions among $72.2 \%$ of enrolled patients. It is worthy to note that most patients that undergo transfusion often suffer from a surplus of iro, which can be fatal and can be overcome with iron chelate therapy.

Around 56,000 conceptions worldwide are caused due to thalassemia. Of this number, about 30,000 people are affected by $\beta$-Thalassemia and about 3,500 people develop hypertensive or $\alpha$-thalassemia syndrome (Kim and Tridane, 2017). It is also estimated that around 9 million people suffering from thalassemia become pregnant every year worldwide. Of these people 1.33 million are at risk of developing severe disorders related thalassemia (Abu-Shaheen et al., 2020; Kim and Tridane, 2017; Weatherall, 2010a).

Thalassemia is a problem in the health sector in many Mediterranean countries, the MENA (Middle East and North Africa), SE (Southeast) Asia, Central Asia and the Indian subcontinent (Weatherall, 2010b).

Gene frequency of $\beta$-thalassemia was found to be lower than $\alpha$-thalassemia and the incidence of $\alpha$ thalassemia reached $25 \%$ in many Southeast Asian regions (Abu-Shaheen et al., 2020). Unlike in the southeast of Asia, countries in the Middle East and North Africa (MENA) region and particularly the Gulf Cooperation Council (GCC) countries including the UAE, have lower prevalence $\alpha$-thalassemia than of $\beta$ thalassemia and carriers (Kim and Tridane, 2017).

The United Arab Emirates (UAE) is the country with the highest presence of thalassemia carrier among all the countries in the Middle Eastern and North African (MENA), due to a huge rate of consanguineous marriage (50\%) (Baysal, 2005; 2011; Denic et al., 2013). This figure has increased and the explanation for this increase is still unknown (Al-Gazali et al., 2005). Thalassemia carriers when present to a high degree is a concern to public health since the chance of having a child suffering from thalassemia major is about one fourth if two thalassemia carriers marry each other thus increasing the over thalassemia incidence rates (Al-Gazali et al., 2005; Baysal, 2005). The government of UAE has made immense efforts to improve awareness of the public and recognize the number of people suffering from Thalassemia. The UAE government, in 2008, launched a campaign nationwide to promote prenatal testing. Premarital testing is mandatory for all couples since 2012 (Dhabi, 2011). Premarital screening provides non-discriminatory genetic counseling to couples who are at-risk (Belhoul et al., 2013). 
Couples carrier for thalassemia can obtain information about the risk of thalassemia in children and the possibility of prenatal and neonatal diagnosis and prenatal diagnosis. However, the final decision about marriage is up to them and many carrier couples still choose to marry. It is also worth noting that abortion for thalassemia is not performed in the UAE as a solution for the prevention of thalassemia. Today the number of affected births has almost halved before prevention was imposed (Daleure and Al Shareef, 2015). However, prevention of thalassemia in the United Arab Emirates still requires promotion of prevention programs, ongoing public awareness and cooperative national genetic services (Al-Gazali et al., 2005).

The direction of all these attempts to prevent thalassemia is based on two hypotheses:

- Premarital screening prevents thalassemia carriers to marry each other

- In addition to premarital screening, inter-carrier marriage is prevented when young adults are educated about thalassemia

Both hypotheses have been put forward by several governments that have to manage thalassemia in their country. The initial hypothesis is that the largest population of thalassemia will be reduced and thalassemia will be excluded from the population. Today, the United Arab Emirates, like many other countries, celebrates an almost thalassemia situation due to a variety of preventive measures (Cao and Kan, 2013).

However, as a relatively young country with a large working population, the UAE population will continue to grow, leading to a growing population of people with thalassemia. This means that the number of people suffering from thalassemia in the United Arab Emirates is steadily increasing. As the carrier population continues to marry, cases of thalassemia are likely to reappear. It is to be noted that in the near future, the UAE government should continue to pay attention to the current level of thalassemia prevention programs (Dhabi, 2011).

\section{Prevention of Thalassemia}

Lifelong treatment is mandatory in the case of Thalassemia. This treatment includes but is not limited to blood transfusion, iron chelation agents and expensive medications. There are other costs as well, such as the cost of medical consultation, diagnosis and laboratory testing and treatment for side effects. Due to the fact that most children with thalassemia are born in countries with low per capita income or low-income families, the high cost of treatment places a heavy financial burden on the family of the patient and society at large (Modell and Darlison, 2008; Sattari et al., 2012). Furthermore, the cost of treatment is rising and this is a significant challenge for governments and providers in health care, so it affects the entire national health system (Sattari et al., 2012). Iron chelation drugs have a significant effect on the cost of treatment. Thus, the healthcare system's burden can be lightened by affordance and effective oral iron chelating agents to extend the lifespan of those patient who receive regular blood transfusion and iron chelating agents. Another effective method of reducing thalassemia is the prophylactic method used to reduce the financial burden in many countries (Modell and Darlison, 2008). It is apparent that prevention is much more cost-effective than long-term treatment. Preventive approaches include the following measures: (i) For couples with affected children, prenatal diagnosis indicates that the risk of recurrence is $25 \%$, thus limiting the family size that may reduce fertility rates and (ii) screening of potential air lines (pedigree) for the general public. In countries where there is a high proportion of kin, population testing is useful for economic sensitivities. This test can be initiated between high-risk couples before marriage. Prenatal testing for diagnosis at the prenatal stage is done in China, Hong Kong, India and most parts of Taiwan, Iran, Singapore and southern Europe (Abu-Shaheen et al., 2020).

In the UAE consanguineous marriages are prevalent at a rate of $50 \%$ and result in a high presence of thalassemia carriers (Al-Dabbagh et al., 2014). This high prevalence points towards public health issues. As if the carrier was married, you have a $25 \%$ probability of having children suffering from thalassemia, which is likely to increase the thalassemia population. In addition, UAE is heterogeneous for various $\beta$-thalassemia. In the UAE, the government launched a campaign through out the nation in 2008 to promote premarital screening that provides non-instructive genetic counseling to non-at-risk couples (Belhoul et al., 2013). The risk pair obtains details about the child's risk of developing thalassemia, as well as making decisions about diagnosis, both prenatal and neonatal. The decision finally made, whether to continue or divorce, after the dangers known results in the hands of the couple. Many couples want to continue their marriage (Belhoul et al., 2013). Pregnancy termination has not been implemented in the UAE as a solution for the prevention of thalassemia. Although compared to before the start of preventive screening programs, the number of births affected by Thalassemia has been cut in half (Kim and Tridane, 2017).

As in many other countries, the UAE celebrates thalassemia free activities and promotes a variety of preventive measures (Cao and Kan, 2013).

With so many workers residing in this country that is relatively new, the number of people with thalassemia will become higher in parallel with the population of the UAE (Kim and Tridane, 2017). This leads to speculation that thalassemia in the UAE will still be prevalent due to the presence of carrier marriages that increase the risk of thalassemia. Kim and Tridane (2017) researched whether 
thalassemia preventive measures that include education of the population and premarital screening as discussed earlier, can lead to eradication of thalassemia in due time in the long term in the UAE. The authors conclude that these education and premarital screening can only reduce the spread of thalassemia in the short term, but they cannot eliminate the presence of the disease in the long run.

Due to the high incidence and the severity of the disease, pioneering Mediterranean countries have provided carrier screening and primary prevention mainly for Thalassemia Major, the most frequent form in the area. The tools used for identifying couples at risk during an informed reproductive choice are information, premarital/preconception screening or screening in early pregnancy (Giordano et al., 2014).

In addition, conducted a survey of 17,826 subjects in the UAE where it was revealed that there was a prevalence of $2.98 \% \beta$-thalassemia and 28 pairs $(0.31 \%)$ of high-risk hemoglobinopathy in the entire study population (Salama and Saleh, 2016).

\section{Conclusion}

Despite various measures of the thalassemia premarital screening program, the premarital screening program has not been able to effectively reduce the frequency of marriages in high-risk couples. However, previous studies have shown that focusing on young people and raising awareness of thalassemia though education of informing the population of its increasing burden may reduce inter-carrier marriage and reduce the presence of such disorders.

\section{Acknowledgement}

We'd like to thank our RAK Medical and Health Sciences University, Ras Al Khaimah, United Arab Emirates, for their encouragement and support.

\section{Author's Contributions}

Pooja Shivappa and Grisilda Vidya Bernhardt: Planned the need for review, edited and finalized the manuscript.

Manal Munir Mufti and Ayesha Banu: Literature review and wrote the draft manuscript.

\section{Ethics}

The Institutional Ethical committee approved the review (RAKMHSU-REC-041-2020/21-UG-M)

\section{References}

Abu-Shaheen, A., Heena, H., Nofal, A., Abdelmoety, D. A., Almatary, A., Alsheef, M., \& AlFayyad, I. (2020). Epidemiology of Thalassemia in Gulf Cooperation Council Countries: A Systematic
Review. BioMed Research International, 2020. https://doi.org/10.1155/2020/1509501

Al-Dabbagh, B., Shawqi, S., Yasin, J., Al Essa, A., Nagelkerke, N., \& Denic, S. (2014). Half of the Emirati population has abnormal red cell parameters: challenges for standards and screening guidelines. Hemoglobin, 38(1), 56-59. https://doi.org/10.3109/03630269.2013.848811

Al-Gazali, L. I., Alwash, R., \& Abdulrazzaq, Y. M. (2005). United Arab Emirates: communities and community genetics. Public Health Genomics, 8(3), 186-196. https://doi.org/10.1159/000086764

Ankra-Badu, G. A., Al-Jama, A., \& Al Kadim, Y. (2001). Hemoglobin $\mathrm{H}$ disease in the Al-Qatif region of Saudi Arabia. Annals of Saudi Medicine, 21(5-6), 308-311. https://doi.org/10.5144/0256-4947.2001.308

Baysal, E. (2005). Molecular heterogeneity of betathalassemia in the United Arab Emirates. Public Health Genomics, 8(1), 35-39. https://doi.org/10.1159/000083336

Baysal, E. (2011). Molecular basis of $\beta$-thalassemia in the United Arab Emirates. Hemoglobin, 35(5-6), 581-588. https://doi.org/10.3109/03630269.2011.634706

Belhoul, K. M., Abdulrahman, M., \& Alraei, R. F. (2013). Hemoglobinopathy carrier prevalence in the United Arab Emirates: first analysis of the Dubai Health Authority premarital screening program results. Hemoglobin, 37(4), 359-368. https://doi.org/10.3109/03630269.2013.791627

Cao, A., \& Galanello, R. (2010). Beta-thalassemia. Genetics in medicine, 12(2), 61-76. https://doi.org/10.1097/GIM.0b013e3181cd68ed

Cao, A., \& Kan, Y. W. (2013). The prevention of thalassemia. Cold Spring Harbor Perspectives in Medicine, 3(2), a011775. https://doi.org/10.1101/cshperspect.a011775

Daleure, G., \& Al Shareef, Z. (2015). Exploring underrepresentation of young Emirati adults in the UAE private sector by examining Emirati job Satisfaction. Sheikh Saud Bin Saor Al Qasimi Foundation for Policy Research. https://doi.org/10.18502/aqf.0114

Danjou, F., Anni, F., \& Galanello, R. (2011). Betathalassemia: from genotype to phenotype. Haematologica, 96(11), 1573. https://doi.org/10.3324/haematol.2011.055962

Denic, S., Aden, B., Nagelkerke, N., \& Essa, A. A. (2013). $\beta$-Thalassemia in Abu Dhabi: consanguinity and tribal stratification are major factors explaining the high prevalence of the disease. Hemoglobin, 37(4), 351-358. https://doi.org/10.3109/03630269.2013.790827

Dhabi, H. A. A. (2011). Health Statistics: 2013: Health Authority Abu Dhabi Abu Dhabi, United Arab Emirates.

Giordano, P. C., Harteveld, C. L., \& Bakker, E. (2014). Genetic epidemiology and preventive healthcare in multiethnic societies: The hemoglobinopathies. International Journal of 
Environmental Research and Public Health, 11(6), 6136-6146. https://doi.org/10.3390/ijerph110606136

Kim, S., \& Tridane, A. (2017). Thalassemia in the United Arab Emirates: why it can be prevented but not eradicated. PLoS ONE, 12(1), e0170485. https://doi.org/10.1371/journal.pone.0170485

Modell, B., \& Darlison, M. (2008). Global epidemiology of haemoglobin disorders and derived service indicators. Bulletin of the World Health Organization, 86, 480-487. https://doi.org/10.2471/BLT.06.036673

Salama, R. A. A., \& Saleh, A. K. (2016). Effectiveness of premarital screening program for thalassemia and sickle cell disorders in Ras Al Khaimah, United Arab Emirates. Journal of Genetic Medicine, 13(1), 26-30. https://doi.org/10.5734/JGM.2016.13.1.26

Sattari, M., Sheykhi, D., Nikanfar, A., Pourfeizi, A. H., Nazari, M., Dolatkhah, R., \& Mashayekhi, S. (2012). The financial and social impact of thalassemia and its treatment in Iran. Pharmaceutical Sciences, 18(3), 171-176.
Vichinsky, E. P. (2005). Changing patterns of thalassemia worldwide. Annals of the New York Academy of Sciences, 1054(1), 18-24. https://doi.org/10.1196/annals.1345.003

Weatherall, D. (2010a). The inherited diseases of hemoglobin are an emerging global health burden. Blood, 115(22), 4331-4336.

https://doi.org/10.1182/blood-2010-01-251348

Weatherall, D. (2010b). Thalassemia as a global health problem: recent progress toward its control in the developing countries. Annals of the New York Academy of Sciences, 1202(1), 17-23. https://doi.org/10.1111/j.1749-6632.2010.05546.x 